\title{
Joint Channel Estimation with Phase Noise Suppression and Soft Decision Decoding Scheme for OFDM-based WLANs
}

\author{
Yong-Hwa Kim and Seong-Cheol Kim \\ School of Electrical Engineering and Computer Science \\ Seoul National University \\ Seoul, Korea \\ \{yhkim,sckim\}@maxwell.snu.ac.kr
}

\begin{abstract}
In orthogonal frequency-division multiplexing (OFDM)-based wireless local area networks (WLANs), phase noise (PHN) and channel estimation errors can degrade the performance of the system. This letter provides a soft decision decoding scheme analysis for OFDM-based WLANs in the presence of PHN and channel estimation errors. Basing on this analysis, we propose a novel iterative scheme for joint channel estimation with PHN suppression and soft decision decoding. In addition, the soft decision decoding metric for QAM OFDM systems is modified to mitigate the effects of PHN and channel estimation errors. The simulation results show that the proposed scheme mitigates the performance degradation due to PHN and channel estimation errors effectively.
\end{abstract}

Keywords- Orthogonal frequency-division multiplexing (OFDM), quadrature amplitude modulation (QAM), channel estimation, phase noise (PHN), wireless local area networks (WLANS).

\section{INTRODUCTION}

Orthogonal frequency-division multiplexing (OFDM) has recently received considerable attention for its robustness against frequency selective multi-path channels. It has been adopted by the IEEE $802.11 \mathrm{a}$ standard as the transmission technique for high -rate wireless local area networks (WLANs) [1]. The packet of OFDM-based WLANs consists of an OFDM packet preamble, a SIGNAL field, and an OFDM DATA field. The packet preamble included ten short pilots and two long pilots which are used to perform the timing/frequency synchronization and channel estimation. The OFDM DATA field is composed of multiple OFDM symbols whose data bits are convolutionally encoded to exploit frequency diversity in frequency selective channels. Li and Liu suggested a sequential method for the estimation of carrier frequency offset, symbol timing and channel response by exploiting the structure of the packet preamble specified by the IEEE 802.11a standard in [2], in which the estimates of decoded symbols are used to improve the channel estimation accuracy.

However, as the literature has reported, the OFDM system is very sensitive to phase noise (PHN) [3]-[7]. PHN can cause the common phase error (CPE) and the inter-carrier interferences (ICI), which seriously degrade the performance of OFDM-based WLANs. Under perfect channel estimation, BarNess and $\mathrm{Wu}$ proposed decision-directed phase noise suppression (PNS) algorithms for the OFDM system in [5], [6] and the effect of PHN on coded OFDM transmission is investigated in [7]. This letter analyzes the performance degradation of soft decision decoding schemes for OFDMbased WLANs in the presence of PHN and channel estimation errors. Then, we propose a new iterative scheme for joint channel estimation with PHN suppression and soft decision decoding which uses pilot sub-carriers, null sub-carriers and the soft estimates of decoded symbols. In order to mitigate the effects of PHN and channel estimation errors, we modify the soft decision decoding metric for QAM OFDM systems using the generalized log likelihood ratio (GLLR) metric which is proposed for QAM symbols under the assumption of channel estimation errors [8]. It will be shown to be highly effective in combating PHN and channel estimation errors for OFDMbased WLANs.

The rest of this paper is organized in the following order. Section II describes the system model of an OFDM system with PHN. Section III describes the proposed joint channel estimation with PHN suppression and soft decision decoding scheme. Section IV presents the computer simulation results in terms of bit error rate (BER) performance of the proposed algorithm over a frequency selective fading channel. Some conclusion remarks are given in section $\mathrm{V}$.

\section{SYSTEM MODEL}

The transmitter of the OFDM system considered in this letter is shown in Fig. 1(a). A finite sequence of binary uncoded bits $u_{a}$ is convolutionally encoded with a coding rate $1 / 2$. Coded bits are segmented into $L$ blocks of length $N_{C B P S}$ (corresponding to the number of coded bits per data OFDM symbol), which are interleaved by a block channel interleaver [1]. Interleaved coded bits in each block are grouped and mapped to one of the $2^{M}$ possible complex symbols in the quadrature amplitude modulation (QAM) constellation $S$. 


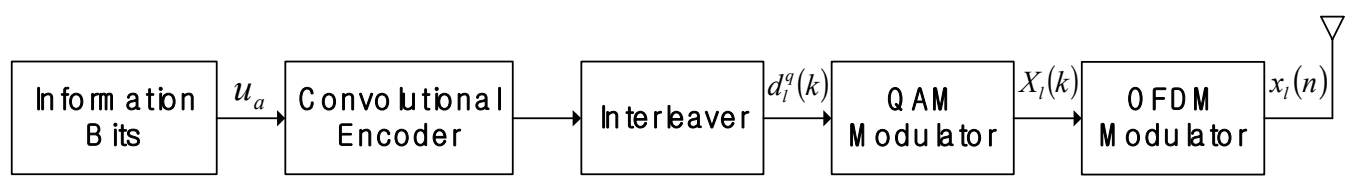

(a) Transmitter

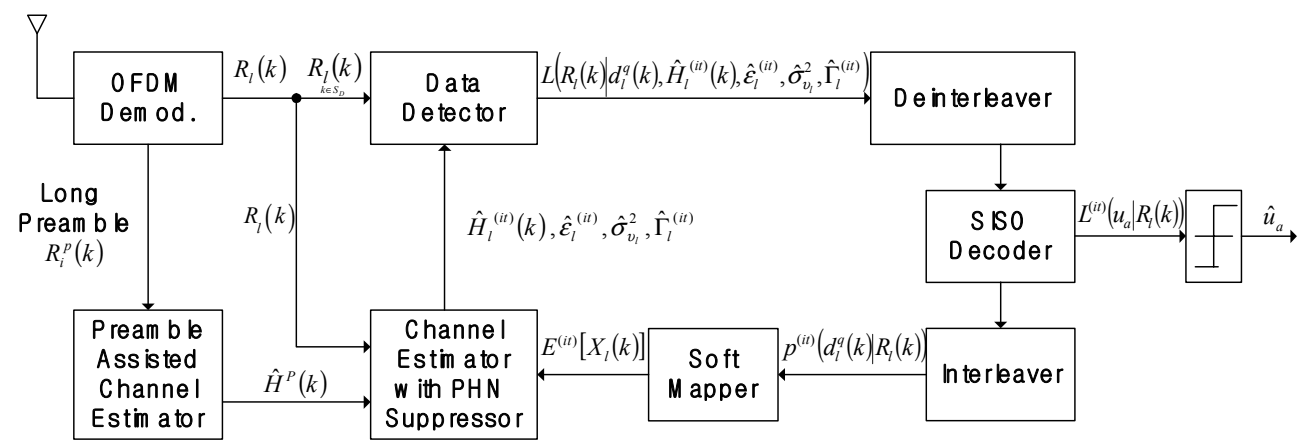

(b) Receiver

Fig. 1 OFDM system model with iterative scheme for joint channel estimation with PHN suppression and soft decision decoding

Complex symbols are used to modulate corresponding subcarriers. We can simply express this mapping function for the $k$ th frequency-domain sub-carrier of the $l$ th data OFDM symbol as $X_{l}(k)=\Lambda\left(d_{l}^{0}(k), d_{l}^{1}(k), \cdots, d_{l}^{M-1}(k)\right)$ where an interleaved coded bit $d_{l}^{q}(k)(q=0, \cdots, M-1)$ is the $q$ th bit mapped to a $2^{M}$-QAM symbol $X_{l}(k) \in S$ and the mapping between $X_{l}(k)$ and $\left(d_{l}^{0}(k), \cdots, d_{l}^{M-1}(k)\right)$ is one to one. Each data OFDM symbol is transmitted by $N=64$ sub-carriers being composed of data sub-carrier set $S_{D}$ with $N_{D}=48$ sub-carriers, pilot sub-carrier set $S_{P}$ with $N_{P}=4$ sub-carriers and null subcarrier set $S_{N}$ with $N_{N}=12$ sub-carriers. The time-domain data OFDM symbol in the OFDM data field is generated by the IDFT and the cyclic prefix (CP) of length $N_{c p}$ is added at the end of each time-domain data OFDM symbol.

It is assumed that the timing and frequency synchronization is perfect by the packet preamble. The $n$th sample of the received time-domain signal can be expressed by

$$
r_{l}(n)=\left[x_{l}(n) \otimes h_{l}(n)\right] e^{j \theta_{l}(n)}+w_{l}(n)
$$

where $x_{l}(n), \otimes, h_{l}(n), \theta_{l}(n)$ and $w_{l}(n)$ denote the transmitted signal, the convolution, the channel impulse response, phase noise (PHN) and the additive white Gaussian noise (AWGN), respectively. We assume that the maximum delay of the channel impulse response is shorter than the CP length $N_{c p}$. $\mathrm{PHN}$ is generated by both transmitter and receiver oscillators and can be modeled as a discrete time Wiener process [6]. After removing the $\mathrm{CP}$ and taking the DFT, the resulting frequency-domain signal $R_{l}(k)$ can be obtained by

$$
R_{l}(k)=\varepsilon_{l} X_{l}(k) H_{l}(k)+\xi_{l}(k)+W_{l}(k)
$$

where $H_{l}(k)$ and $W_{l}(k)$ denote the frequency-domain expression of $h_{l}(n)$ and $w_{l}(n)$, respectively. Moreover, the common phase error (CPE) $\varepsilon_{l}$ and the inter-carrier interference (ICI) $\xi_{l}(k)$ in (2) caused by PHN can be given by following equations:

$$
\begin{gathered}
\varepsilon_{l}=\frac{1}{N} \sum_{n=0}^{N-1} e^{j\left(\theta_{l}(n)\right)}, \\
\xi_{l}(k)=\sum_{\substack{m=-N / 2 \\
m \neq k}}^{N / 2-1} \frac{X_{l}(m) H_{l}(m)}{N} \sum_{n=0}^{N-1} e^{j\left(\frac{2 \pi(m-k) n}{N}+\theta_{l}(n)\right)} .
\end{gathered}
$$

The transmitted symbol $X_{l}(k)$ is assumed as a random variable independent of $H_{l}(k)$ with zero mean and variance $\sigma_{X}^{2}$. The ICI $\xi$ in (4) can be approximated as a zero mean Gaussian random variable with variance $\sigma_{\xi}^{2}$ [4]. For the simplicity of the calculation, we can assume that the auto-correlation of channel impulse response $\mathrm{E}\left[\left|H_{l}(k)\right|^{2}\right]$ is equal to 1 . The normalized ICI variance $\sigma_{\xi}^{2} / \sigma_{X}^{2}$ can be approximated by $(2 \pi \beta T) \cdot\left(N-N_{N}\right) / 3 N$ where $T$ denotes the OFDM symbol period [5], and the parameter $\beta$ is well described in [3]. Here, we define the ICI-plus-noise as $v_{l}(k)=\xi_{l}(k)+W_{l}(k)$ which has zero mean and variance $\sigma_{v_{l}}^{2}=\sigma_{\xi_{l}}^{2}+\sigma_{W_{l}}^{2}$.

\section{ITERATIVE Joint Channel Estimation With Phase} NoISE SuPpression AND SOFT DECISION DECODING SCHEME

Fig. 1(b) shows a block diagram of the proposed joint channel estimation with PHN suppression and soft decision decoding (JCPS) scheme. To obtain the channel coefficient estimates, we use two pilot symbols in the long preamble. The estimated channel response of the $i$ th pilot symbol in the long preamble can be obtained by

$$
\hat{H}_{i}^{p}(k)=R_{i}^{p}(k) / P_{i}(k)=\varepsilon_{i} H_{i}(k)+\underbrace{\left(\xi_{i}(k)+W_{i}(k)\right) / P_{i}(k)}_{e_{i}(k)}
$$


where $R_{i}^{p}(k), P_{i}(k)$ and $e_{i}(k)$ denote the received pilot, the known transmitted pilot and the channel estimation error, respectively. An initial channel estimate can be obtained by

$$
\hat{H}_{l}^{(i t=0)}(k)=\left(\sum_{i=0}^{1} \hat{H}_{i}^{p}(k)\right) / 2
$$

where it denotes the iteration index. The CPE $\varepsilon$ in (3) is assumed to be $e^{j \Phi}$ since $|\mathcal{E}| \approx 1$. To obtain the phase estimates of the CPE, the least-square (LS) method is applied to minimize the cost function

$$
\hat{\Phi}_{l}=\arg \min _{\Phi_{l}} \sum_{k}\left|R_{l}(k)-e^{j \Phi_{l}} X_{l}(k) \hat{H}_{l}(k)\right|^{2}
$$

which leads to the estimate $\hat{\Phi}_{l}$ given by the following equation:

$$
\hat{\Phi}_{l}=-\measuredangle\left(\sum_{k} R_{l}^{*}(k) X_{l}(k) \hat{H}_{l}(k)\right) .
$$

where $\angle(x)$ denotes the complex angle of $x$. Using the pilot sub-carriers at every data OFDM symbol, the initial CPE estimate $\hat{\varepsilon}_{l}^{(i t=0)}=e^{j \hat{\Phi}_{l}^{(i t=0)}}$ is estimated by

$$
\hat{\Phi}_{l}^{(i t=0)}=-\measuredangle\left(\sum_{k \in S_{P}} R_{l}^{*}(k) X_{l}(k) \hat{H}_{l}^{(i t=0)}(k)\right) .
$$

Using the null sub-carriers, the estimate of the ICI-plus-noise energy can be given by [5], [6]

$$
\hat{\sigma}_{v_{l}}^{2}=\frac{1}{N_{N}} \sum_{k \in S_{N}}\left|R_{l}(k)\right|^{2} .
$$

Given $\hat{H}_{l}^{(i t)}(k), \hat{\varepsilon}_{l}^{(i t)}$ and $\hat{\sigma}_{v_{l}}^{2}$, the conventional minimum distance log-likelihood ratios (LLRs) of conditional probabilities on the coded bits can be calculated with the maxlog approximation as [8]

$$
\begin{aligned}
& L^{(i t)}\left(R_{l}(k) \mid d_{l}^{q}(k), \hat{H}_{l}{ }^{(i t)}(k), \hat{\varepsilon}_{l}{ }^{(i t)}, \hat{\sigma}_{v_{l}}^{2}\right) \\
& =\log \frac{\operatorname{Pr}\left(R_{l}(k) \mid d_{l}^{q}(k)=+1, \hat{H}_{l}{ }^{(i t)}(k), \hat{\varepsilon}_{l}{ }^{(i t)}, \hat{\sigma}_{v_{l}}^{2}\right)}{\operatorname{Pr}\left(R_{l}(k) \mid d_{l}^{q}(k)=-1, \hat{H}_{l}{ }^{(i t)}(k), \hat{\varepsilon}_{l}{ }^{(i t)}, \hat{\sigma}_{v_{l}}^{2}\right)} \\
& \approx \frac{1}{\hat{\sigma}_{v_{l}}^{2}}\left(\max _{X_{l}(k)=D_{+1}^{q}}\left\{-\left|R_{l}(k)-\hat{\varepsilon}_{l}{ }^{(i t)} X_{l}(k) \hat{H}_{l}{ }^{(i t)}(k)\right|^{2}\right\}\right. \\
& \left.\quad-\max _{X_{l}(k) \in D_{-1}^{q}}\left\{-\left|R_{l}(k)-\hat{\varepsilon}_{l}{ }^{(i t)} X_{l}(k) \hat{H}_{l}{ }^{(i t)}(k)\right|^{2}\right\}\right)
\end{aligned}
$$

where $D_{ \pm 1}^{q}$ denotes a set of symbols $X_{l}(k)$ for which $d_{l}^{q}(k)= \pm 1$. However, when channel estimation errors exist, the conventional minimum distance metric gives a fairly poor performance for QAM demodulation [8]. The proposed generalized LLRs (GLLRs) of conditional probabilities on the coded bits can be calculated as

$$
\begin{aligned}
& L^{(i t)}\left(R_{l}(k) \mid d_{l}^{q}(k), \hat{H}_{l}^{(i t)}(k), \hat{\varepsilon}_{l}^{(i t)}, \hat{\sigma}_{v_{l}}^{2}, \hat{\Gamma}_{l}^{(i t)}\right) \\
& \approx \frac{1}{\hat{\sigma}_{v_{l}}^{2}}\left\{\max _{X_{l}(k) \in D_{+1}^{q}}\left\{-\frac{\left|R_{l}(k)-\hat{\varepsilon}_{l}^{(i t)} X_{l}(k) \hat{H}_{l}^{(i t)}(k)\right|^{2}}{\left|X_{l}(k)\right|^{2} \hat{\Gamma}_{l}^{(i t)}+1}\right\}\right. \\
& -\max _{X_{l}(k) \in D_{-1}^{q}}\left\{-\frac{\left|R_{l}(k)-\hat{\varepsilon}_{l}^{(i t)} X_{l}(k) \hat{H}_{l}^{(i t)}(k)\right|^{2}}{\left|X_{l}(k)\right|^{2} \hat{\Gamma}_{l}^{(i t)}+1}\right\}
\end{aligned}
$$

where $\hat{\Gamma}_{l}^{(i t)}=\hat{\sigma}_{e_{l}}^{2(i t)} / \hat{\sigma}_{v_{l}}^{2}$. The initial channel estimation error $e_{l}^{(i t=0)}(k)=\left(\sum_{i=0}^{1} e_{i}(k)\right) / 2 \quad$ with variance $\hat{\sigma}_{e_{l}{ }^{(i t=0)}}^{2}=\left(\hat{\sigma}_{\xi}^{2}+\hat{\sigma}_{w}^{2}\right) / 2$ is the sum of two independent random variables the ICI and the AWGN. Then, in the practical application, the initial estimates of $\Gamma$ can be obtained by

$$
\hat{\Gamma}_{l}^{(i t=0)}=\left(\frac{1}{2} \cdot \frac{1}{(l+1)} \sum_{j=0}^{l} \hat{\sigma}_{v_{j}}^{2}\right) / \hat{\sigma}_{v_{l}}^{2} .
$$

After deinterleaving the interleaved LLRs in (12) on the coded bits, soft-input soft-output (SISO) decoder generates LLRs of a posteriori probabilities on the uncoded bits $L^{(i t)}\left(u_{a} \mid R_{l}(k)\right)$ and those on the coded bits [9]. Hard decisions on the uncoded bits $u_{a}$ are determined using the LLRs of $a$ posteriori probabilities on the uncoded bits $L^{(i t)}\left(u_{a} \mid R_{l}(k)\right)$.

However, the initial channel estimates may not be accurate because of the ICI-plus-noise and the CPE. The CPE has a different value at each pilot symbol in the long preamble. Under imperfect channel estimation, the initial CPE estimation obtained by limited numbers of pilot sub-carriers may not be accurate. Meanwhile, after decoding the entire packet, we can use the expected value of the transmitted symbol to improve the accuracy of the CPE estimation and the channel estimation. After interleaving the deinterleaved LLRs on the coded bits from SISO decoder, we use the LLRs of a posteriori probabilities on the coded bits $L^{(i t)}\left(d_{l}^{q}(k) \mid R_{l}(k)\right)$ to calculate the expected value of the transmitted symbol $E^{(i t)}\left[X_{l}\right.$ $(k)$ ] by

$$
E^{(i t)}\left[X_{l}(k)\right]=\sum_{X_{l}(k) \in S} X_{l}(k) \cdot p^{(i t)}\left(X_{l}(k) \mid R_{l}(k)\right)
$$

where $\quad p^{(i t)}\left(X_{l}(k) \mid R_{l}(k)\right)=\prod_{q=(0, \cdots, M-1)} p^{(i t)}\left(d_{l}^{q}(k) \mid R_{l}(k)\right) \quad$. $p^{(i t)}\left(d_{l}^{q}(k) \mid R_{l}(k)\right)$ for $d_{l}^{q}(k)= \pm 1$ can be obtained by

$$
\begin{aligned}
& p^{(i t)}\left(d_{l}^{q}(k)=+1 \mid R_{l}(k)\right)=\frac{\exp \left(L^{(i t)}\left(d_{l}^{q}(k) \mid R_{l}(k)\right)\right)}{1+\exp \left(L^{(i t)}\left(d_{l}^{q}(k) \mid R_{l}(k)\right)\right)}, \\
& p^{(i t)}\left(d_{l}^{q}(k)=-1 \mid R_{l}(k)\right)=\frac{1}{1+\exp \left(L^{(i t)}\left(d_{l}^{q}(k) \mid R_{l}(k)\right)\right)} .
\end{aligned}
$$

Using the expected value of the transmitted symbol, the CPE is estimated as 


$$
\hat{\Phi}_{l}^{(i t)}=-\measuredangle\left(\sum_{k \in S_{D}} R_{l}^{*}(k) E^{(i t-1)}\left[X_{l}(k)\right] \hat{H}_{l}^{(i t-1)}(k)\right) .
$$

After the CPE estimation by (16), we determine whether or not the expected value of the transmitted symbol at each OFDM symbol associated with $X_{l}(k)$ will be used for the channel estimation. If the sum of distances $d_{l}$ at each OFDM symbol is below the threshold $t h$, we replace the channel estimates with

$$
\begin{aligned}
\hat{H}_{l}^{(i t)}(k) & =\frac{2}{2+\beta} \hat{H}_{l}^{(i t-1)}(k) \\
& +\frac{\beta}{2+\beta}\left(e^{j \hat{\Phi}_{l}^{(i t)}} E^{(i t-1)}\left[X_{l}(k)\right]\right)^{*} R_{l}(k)
\end{aligned}
$$

where $d_{l}=\sum_{k \in S_{D}}\left|R_{l}(k)-e^{j \hat{\Phi}_{l}^{(i t)}} E^{(i t-1)}\left[X_{l}(k)\right] \hat{H}_{l}^{(i t-1)}\right|^{2}$ is the sum of distances, the threshold th equals $96 \hat{\sigma}_{v_{l}}^{2}$ and $\beta=\mid E^{(i t-1)}\left[X_{l}(k)\right]^{2}$ is a weighting factor [2]. It is very hard to determine the estimation error of $\hat{H}_{l}^{(i t)}(k)$, since it depends on $e^{j \hat{\Phi}_{l}^{(i t)}}, E^{(i t-1)}\left[X_{l}(k)\right]$ and $R_{l}(k)$. Therefore, the estimate of $\Gamma$ given by

$$
\hat{\Gamma}_{l}^{(i t)}=\frac{4}{9} \cdot\left(\sum_{l=0}^{L-1} \hat{\Gamma}_{l}^{(i t-1)} / L\right)
$$

is used in (12) as a parameter for the estimation error of $\hat{H}_{l}{ }^{(i t)}(k)$.

The proposed GLLR-based JCPS scheme is implemented by the following steps.

Step 1) Set $i t$ to zero. Estimate the initial channel $\hat{H}_{l}^{(i t=0)}(k)$ by (6), the initial $\operatorname{CPE} \hat{\varepsilon}_{l}^{(i t=0)}$ by (9), the ICI-plus-noise energy $\hat{\sigma}_{v_{l}}^{2}$ by (10) and the initial $\hat{\Gamma}_{l}^{(i t=0)}$ by (13).

Step 2) Use (12) to calculate the LLRs of conditional probabilities on the coded bits with $\hat{H}_{l}^{(i t)}(k), \hat{\varepsilon}_{l}^{(i t)}, \hat{\sigma}_{v_{l}}^{2}$ and $\hat{\Gamma}_{l}^{(i t)}$.

Step 3) After deinterleaving, generate the LLRs of a posteriori probabilities on the uncoded bits $L^{(i t)}\left(u_{a} \mid R_{l}(k)\right)$ and those on the coded bits by SISO decoder. Iteration continues until the BER saturates. After interleaving, calculate the expected value of the transmitted symbol $E^{(i t)}\left[X_{l}(k)\right]$ by (14), and go to Step 4) with $i t \leftarrow i t+1$.

Step 4) Estimate the CPE $\hat{\varepsilon}_{l}^{\left({ }^{(i t)}\right.}$ by (16). If $d_{l}$ at each OFDM symbol is below th, replace the channel estimates by (17) and $\hat{\Gamma}_{l}^{(i t)}$ by (18). Otherwise, we maintain the channel estimates $\hat{H}_{l}^{(i t)}(k)=\hat{H}_{l}^{(i t-1)}(k)$ and $\hat{\Gamma}_{l}^{(i t)}=\hat{\Gamma}_{l}^{(i t-1)}$. And go to Step 2).

\section{Simulation Results}

The convolutional code chosen in this letter is a rate $1 / 2$ recursive systematic convolutional (RSC) encoder $\left(g_{1}=5\right.$ and $g_{2}=7$ in octal). The interleaver and tail puncturing follow specification in [1]. The OFDM system data subcarriers are modulated by 16 QAM $(M=4)$. The OFDM symbol period $T$ is $4.0 \mu \mathrm{s}$ and the number of OFDM data symbols within a packet $L$ is 20 . The average signal-to-noise ratio (SNR) is $\left(52 \sigma_{X}^{2}\right) /\left(64 \sigma_{W}^{2}\right)$. The PA and the PCE represent the long preamble assisted channel estimation and the perfect channel estimation, respectively. It is assumed that frequency selective fading channels have six multipaths with exponentially decaying power delay profile [5] and are static within a packet.

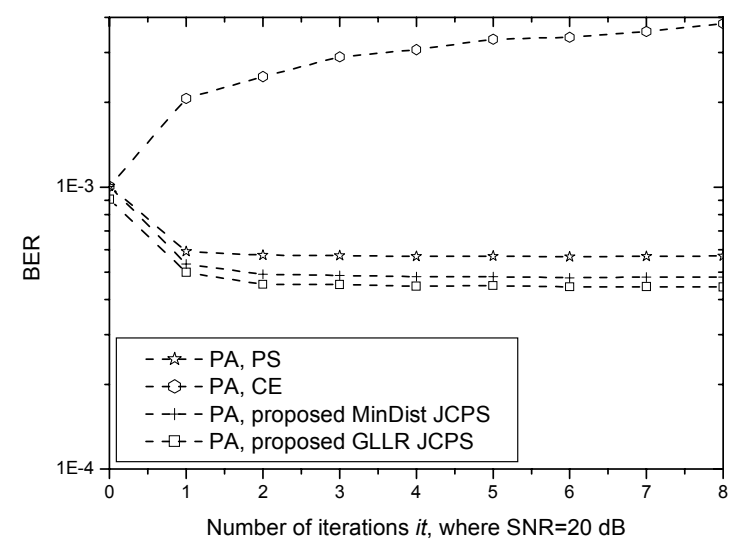

Fig. 2 BER performance versus the number of iterations with PHN energy $4 \pi \beta T$ equal to $0.0384 \mathrm{rad}^{2}$

Fig. 2 depicts the convergence properties of the proposed joint channel estimation with PHN suppression and soft decision decoding (JCPS) schemes at $\mathrm{SNR}=20 \mathrm{~dB}$. It is seen that the one iteration $(i t=1)$ is enough to reach the converged bit error rate (BER) performance. Therefore, we fix the number of iterations to one $(i t=1)$ in the following simulation results. Furthermore, GLLR-based JCPS scheme shows a little improvement in the BER performance compared with the conventional minimum distance metric (MinDist) based JCPS scheme. JCPS schemes give the better BER performance than iterative schemes only with the PHN suppression algorithm (PS) by (16) and only with the channel estimation algorithm (CE) by (17).

Fig. 3 shows the BER performance evaluated by computer simulations with PHN energy $4 \pi \beta T$ equal to $0.0384 \mathrm{rad}^{2}$. From this figure, the proposed GLLR-based JCPS scheme outperforms soft decision decoding schemes with the phase noise suppression (PNS) algorithms using $\hat{\varepsilon}_{l}$ and $\hat{\sigma}_{v_{l}}^{2}$ in [5]. The BER performance of the GLLR-based JCPS scheme is almost the same as the BER performance curve for PA without PHN and shows $1.9 \mathrm{~dB}$ gain loss from the BER performance curve for PCE without PHN. 


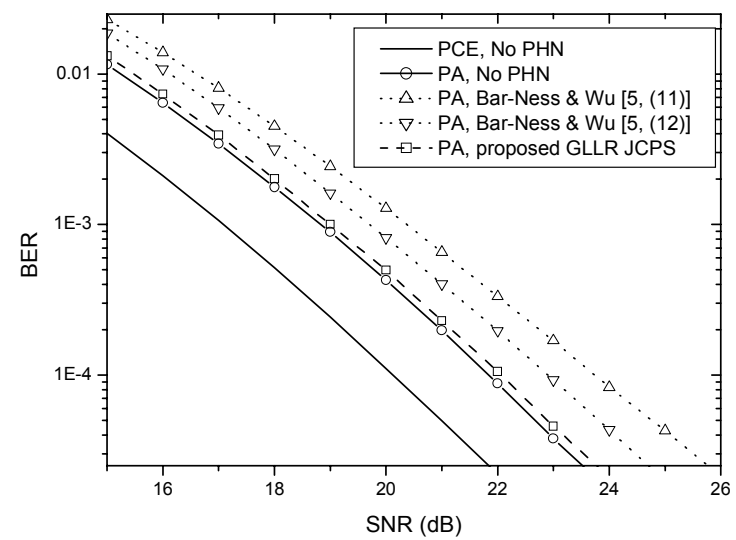

Fig. 3 BER performance with PHN energy $4 \pi \beta T$ equal to 0.0384 $\operatorname{rad}^{2}$

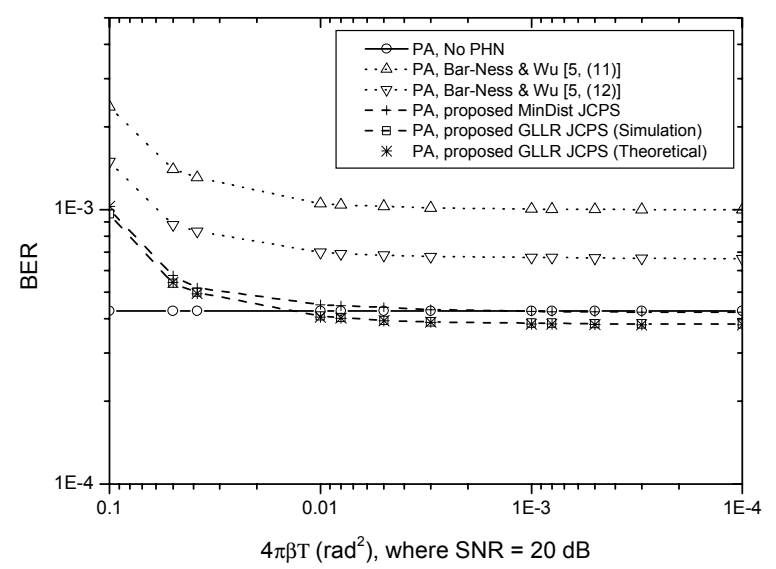

Fig. 4 BER performance with phase noise energy $4 \pi \beta T$

Fig. 4 presents the BER performance with PHN energy at $\mathrm{SNR}=20 \mathrm{~dB}$. It is seen that, for various PHN conditions, the performance of the proposed GLLR-based JCPS scheme always outperforms soft decision decoding schemes with the PNS algorithms using $\hat{\varepsilon}_{l}$ and $\hat{\sigma}_{v_{l}}^{2}$ in [5] and conventional MinDist-based JCPS scheme proposed in this letter. Furthermore it is close to the GLLR-based JCPS scheme with the exact ICI-plus-noise variance $\sigma_{v}^{2}$. From Fig. 4, if PHN energy $4 \pi \beta T$ is in the order of $10^{-2}$ or lower, the proposed
GLLR-based JCPS scheme gives the better performance with positive gain than the BER performance curve for PA without PHN.

\section{CONCLUSION}

In this letter, we proposed the GLLR-based joint channel estimation with PHN suppression and soft decision decoding (JCPS) scheme for OFDM-based WLANs. The proposed scheme obtains performance improvement using pilot subcarriers, null sub-carriers and decoded data. Simulation results have been shown that the proposed scheme has much better performance than other soft decision decoding schemes under imperfect channel estimation. This scheme can be further extended to other coded OFDM systems.

\section{ACKNOWLEDGMENT}

This work was supported by University IT Research Center Project.

\section{REFERENCES}

[1] IEEE 802.11 WG, part 11: Wireless LAN Medium Access Control (MAC) and Physical Layer (PHY) Specifications : High-speed Physical Layer in the $5 \mathrm{GHz}$ band, Supplement to IEEE 802.11 Standard, Sep. 1999.

[2] J. Liu, and J. Li, "Parameter estimation and error reduction for OFDM-based WLANs," IEEE Trans. Mobile Computing, vol. 3, no. 2, pp. 152-163, April-June 2004.

[3] T. Pollet, and M. V. Bladel, and M. Moeneclaey, "BER sensitivity of OFDM systems to carrier frequency offset and Wiener phase noise," IEEE Trans. Commun., vol. 43, no. 234, pp. 191-193, Feb./March/April 1995.

[4] A. G. Armada, "Understanding the effects of phase noise in orthogonal frequency division multiplexing," IEEE Trans. Broadcast., vol. 47, no. 2, pp. 153-159, June 2001.

[5] S. Wu, and Y. Bar-Ness, "A phase noise suppression algorithm for OFDM-based WLANs," IEEE Commun. Lett., vol. 6, no. 12, pp. 535-537, Dec. 2002.

[6] S. Wu, and Y. Bar-Ness, "OFDM systems in the presence of phase noise: consequences and solutions," IEEE Trans. Commun., vol. 52, no. 11, pp. 1988-1996, Nov. 2004.

[7] D. Petrovic, W. Rave, and G. Fettweis, "Performance degradation of coded-OFDM due to phase noise," in Proc. IEEE $V T C$, vol. 2, Jeju, April 2003, pp. 1168-1172.

[8] M. M. Wang, W. Xiao, and T. Brown, "Soft decision metric generation for QAM with channel estimation error," IEEE Trans. Commun., vol. 50, no. 7, pp. 1058-1061, July 2002.

[9] S. Benedetto, D. Divsalar, G. Montorsi and F. Pollara, "A softinput soft-output APP module for iterative decoding of concatenated codes" IEEE Commun. Lett., vol. 1, no. 1, pp. 2224, Jan. 1997. 